\title{
Pevista
}

ISSN-L: 2215-4728

DOI: http://dx.doi.org/10.15359/rp.23.5

http://www.revistas.una.ac.cr/perspectivas

N. ${ }^{\circ}$ 23. Julio-Diciembre, 2021 • pp. 1-19

\section{Vivir hoy en una sociedad que históricamente ha sido construida sobre la violación de los derechos humanos. El racismo y el despojo de la humanidad de la población indígena en Guatemala}

\author{
Living Today in a Society that has Historically been \\ Built on the Violation of Human Rights. Racism and \\ the Dispossession of Humanity of the Indigenous \\ Population in Guatemala
} María Alejandra Medrano Escobar*

Fecha de recepción: 14/03/2021 • Fecha de aceptación: 03/05/2021

Resumen: sin lugar a duda, las crisis económicas, políticas o sociales generadas a raíz de la pandemia COVID-19, han sido más drásticas en las sociedades con mayores índices de pobreza, discriminación, racismo, polarización y desigual repartición de la riqueza, Guatemala es un ejemplo concreto
Abstract: The economic, political or social crises because of the COVID-19 pandemic have been more drastic in societies with higher rates of poverty, discrimination, racism, polarization, and unequal distribution of wealth. Guatemala is a concrete example of this situation. Historically it has been

* Guatemalteca. Magíster en Políticas Públicas por la Universidad Rafael Landívar (URL), Facultad de Ciencias Políticas y Sociales, Ciudad de Guatemala, Guatemala. Historiadora por la Universidad de San Carlos de Guatemala (USAC), Escuela de Historia, Ciudad de Guatemala, Guatemala. Docente en la Facultad de Ciencias Políticas y Sociales, Universidad Rafael Landívar (URL), Facultad de Ciencias Políticas y Sociales, Ciudad de Guatemala, Guatemala. Profesora titular y coordinadora de Planificación en la Escuela de Historia (USAC), Ciudad de Guatemala, Guatemala. Correos electrónicos: mamedrano@url.edu.gt, maria.medrano@profesor.usac.edu.gt ORCID: https://orcid.org/0000-0002-6933-4370 
de esta situación. Históricamente, se ha cimentado sobre la miseria, el despojo, la exclusión y el acaparamiento de la riqueza en pocas manos. En este caso, el debate se concentra en una categoría, la de despojo de humanidad, ubicada en el imaginario de una nación que se construyó sobre las bases de racismo y la ciudadanía diferenciada. Esto, por supuesto, es parte de una tradición de violaciones a los derechos humanos, una situación estructural que, en la historia de nación, debe comprenderse como condició de vida de un considerable porcentaje de la población. En las coyunturas actuales, esta herencia histórica define las relaciones que vive la sociedad.

Palabras claves: despojo; racismo; pobreza violación de los derechos humanos; historia. founded on misery, dispossession, exclusion, and the hoarding of wealth in a few hands. In this case, the article focuses on one category, that of dispossession of humanity, located in the imaginary of a nation that was built on the foundations of racism and differentiated citizenship. This, of course, is part of a tradition of Human Rights violations, a structural situation that, in the history of the nation, must be understood as a condition of life for a considerable percentage of the population. In the current time, this historical heritage defines the relationships inside the society.

Keywords: dispossession; racism; poverty; violation of Human Rights; history.

\section{Introducción}

Cuando considerarse persona con todos sus derechos es un privilegio, las consecuencias se transforman en estructuras de larga duración en el nivel económico, político y social, situaciones como esta marcan y definen la historia de países como Guatemala y muchos otros en los que se han construido relaciones y formas de poder desde la marginación, la desigualdad, la inequidad y el despojo (en todo sentido).

¿Qué hacer cuando legalmente una nación se ha construido sobre la premisa de que no todas las personas son iguales? O que algunas, tienen más derechos que las otras, que hay ciudadanos de primera y segunda categoría o que, carga con un pasado histórico en el que se demuestra cómo no todos fueron considerados personas.

Según UNICEF (2020), el problema mencionado continúa definiendo el presente de Guatemala, en esta ocasión, hablar de este tema, nos ayudará a comprender cómo las diversas crisis alrededor de la pandemia COVID-19 que se manifestaron con un grave aumento de la pobreza y la pobreza extrema, también tuvieron una base racista. En
2020, Guatemala ocupó el sexto lugar de desnutrición crónica a nivel mundial y el primer lugar en América Latina, debido a los altos niveles de miseria, de marginación y de desnutrición $2020 .{ }^{1}$

$\mathrm{Si}$ las condiciones de extrema pobreza ya eran graves antes de marzo de 2020, en el país, desde el primer mes de pandemia aparecieron en cada espacio conocido, personas con banderas blancas, muriendo de hambre, en abandono, sin apoyo gubernamental. El Estado inició el proceso de respuesta ${ }^{2}$ hacia la pandemia y la crisis, sin embargo, en ese primer mes, llegaron también (voces que han ido en aumento y aún no se callan en el presente) las crecientes críticas hacia las gestiones que se hacían, empezamos a escuchar los reclamos de corrupción, empezaron a ser cada vez más visibles los robos descarados en cada ministerio o secretaría estatal y la falta de una infraestructura adecuada para atender las necesidades de salud. Tampoco, se observó una respuesta digna, la nula (o descaradamente deficiente) inversión en la salud e integridad de la población era un hecho observado y criticado por todos.

De acá, vamos a extraer un elemento, presente en muchas miradas desde la academia, relativo a cómo buena parte del desinterés del gobierno se debió al racismo, a esa idea estructurada que se ha sembrado en la sociedad guatemalteca, en la que las áreas rurales y principalmente con presencia indígena, no importan, no deben atenderse.

No es de extrañar, por lo tanto, que, durante la pandemia, se hayan vivido serias acciones de corrupción, robos millonarios, maldad en el establecimiento de una infraestructura digna y funcional para enfrentar la crisis. La alianza criminal del llamado pacto de corruptos $^{3}$ que se ha gestado en la institucionalidad y controla todos los poderes del Estado, hizo todo lo posible para desaparecer miles de millones de quetzales ${ }^{4}$

En datos de 2020, UNICEF registró $49.8 \%$ de niños en Guatemala con desnutrición crónica, significa 1 de cada 2 y $3.4 \%$ de mortalidad infantil.

2 Varias palabras, conceptos o ideas, se colocan en forma cursiva, la cual es propia, para identificar el énfasis que la autora da al significado de estas.

3 Establecido desde los años 1990, por familias, personajes y operadores políticos de las mafias que combinaban intereses oligarcas, empresariales, estatales y del narcotráfico Actualmente, reciclado, en un nuevo grupo heredero o de nuevas de redes familiares que viven de las alianzas mafiosas y la corrupción, operando desde hace cinco o seis años.

4 Las pérdidas y los robos más notorios en 2020 fueron: Q135 millones destinados para obras públicas, Q122 millones encontrados en una residencia y producto de sobornos y coimas de funcionarios públicos, Q20 mil millones en préstamos para atender la pandemia y otros 
que hubiesen podido apoyar en alguna medida a la población en condiciones de miseria. Sin embargo, he ahí, en donde radica el punto esencial de este artículo, en la poca importancia que para el Estado tiene la población guatemalteca, de mayoría indígena, en un Estado racista y genocida.

El indio es el atraso del pueblo, ${ }^{5}$ fue uno de los imaginarios que definió las relaciones de poder en la construcción de la nación en el siglo XIX, lo cual ya era la consecuencia de la idea estructurada de la conquista española indio haragán, ser inferior, que debe ser sometido a una cultura superior.

Comprender las bases y los ejes transversales de estas ideas, significa comprender la esencia de la construcción de una sociedad para otros, una sociedad en la que un fuerte porcentaje de la población solo ha sido considerado un componente más de la patria de otros, una nación en la que el despojo es una de las principales categorías de análisis para comprender las diferencias, las desigualdades y la inequidad en el poder $\mathrm{y}$ ante el desarrollo.

En un texto de Aparicio y Pisarello (2008), se señala que "la sujeción y dependencia de otros, en definitiva, constituye un factor central para determinar las posiciones de vulnerabilidad y de poder en el ejercicio de los deberes y de los derechos" (p. 143).

Esto nos hace pensar en por qué en las coyunturas actuales, al estudiar variadas problemáticas, es necesario dirigir la mirada hacia los grupos que históricamente han estado marcados por la vulnerabilidad como condición de vida y de desarrollo, en donde el eje fundamental ha sido el despojo y la creencia de que le pertenecen a los otros, y, por lo tanto, los otros pueden hacer con ellos lo que quieran.

La teoría de derechos humanos, por tanto, como una construcción necesaria en el presente, que se fundamenta en las bases históricas del país, se debe interesar primero en las consecuencias de que en esta

préstamos que a fin de año se generaron para atender las necesidades de las poblaciones afectadas por las tormentas tropicales de temporada.

5 En los textos de Arturo Taracena, Etnicidad, Estado y Nación en Guatemala, tomos I y II (CIRMA, 2002 y 2004), se analiza el dificultoso proceso de adquisición de la ciudadaní bajo el modelo de la ciudadanía diferenciada y pasiva para los indígenas, en donde, por ejemplo, se podí leer, "todos los habitantes de la mente son ciudadanos los guatemaltecos que tengan una profesión, oficio y propiedades, que les proporcionen medios de subsistir con independencia”. (T/I, p. 173) nación se haya ignorado la vida de grandes grupos de población, a lo largo de varios siglos.

Cuando leemos, premisas como los derechos humanos son generalizables a todas las personas por su mera condición de personas, pero a la vez entendemos que al menos en la historia de Guatemala, aproximadamente el $70 \%$ de la población no tuvieron la condición de personas en tres siglos y la condición de ciudadanos (en su totalidad) la lograron hasta mediados del siglo XX, entonces nos enfrentamos a una grave situación, pues no se trata simplemente de reconocer los derechos antes negados, más bien se trata de analizar todas las consecuencias que esta situación ha traído y que sigue marcando y normando en las relaciones sociales, políticas y económicas de las personas.

El presente está marcado con un $62,9 \%$ de pobreza (cifras oficiales del PNUD en 2015/16), o según cifras de la CEPAL (61\% desde 2014) esta última estima con base en los indicadores anteriores, que en 2020 la pobreza extrema pudo haber aumentado cuatro puntos porcentuales, observando la caída un $25 \%$ en el desempleo y el colapso del $12 \%$ de la economía nacional, en un país en el que $66 \%$ de la población económicamente activa se dedica a la economía informal y en el que este sector experimentó un aumento de $94 \%$ de pobreza (y pobreza extrema), debido a la pandemia.

Por lo tanto, el punto acá es que uno de los principales elementos que debiéramos analizar, es el grave desprecio por la población, en muchas ocasiones, originado por cuestiones racistas.

Los derechos como proceso de reconocimiento y sensibles a las vulnerabilidades históricas

Si como afirma Boaventura de Sousa Santos (2014), los derechos humanos son el lenguaje de la dignidad humana, hoy es válido reconocer que muchos pueblos y naciones carecen en grandes proporciones de poseer una vida digna e integral, pues, sus vidas fueron construidas sobre las bases del despojo, la exclusión, la explotación y la discriminación; y, esto se traduce en injusticia, sufrimiento y desigualdad, pues es un hecho que millones de personas en el mundo no son sujeto de 
derechos humanos, por el contrario, hay miles para quienes hablar de derechos es aún una lejana aspiración.

Como bien señala Santos (2014), aunque ya no se hable de colonialismo hay otras formas históricas que continúan limitando el que se hagan valer los derechos de las personas, el neocolonialismo, el racismo, la xenofobia, el estado de excepción permanente en relación con los terroristas, los trabajadores inmigrantes indocumentados, los solicitantes de asilo, las víctimas de las políticas de austeridad a causa de las formas del capital y otras.

Cuando analizamos realidades como la guatemalteca, en este caso, intentando explicar lo que estamos observando en una coyuntura actual, debemos partir de una base esencial que explique los serios procesos de formación social, a partir del despojo como eje de poder en todas sus dimensiones, es decir en lo económico y lo material, pero también ha sido cultural, social, político y humano, partiendo de ideas y acciones estructuradas, como señalar que los indios no fueron seres humanos o que fueron seres sin alma y que no eran más que ociosos y otra serie de argumentaciones que despojaron de humanidad a grandes grupos de población.

Estos elementos que marcaron los procesos de conquista y colonización fueron las bases de cambios drásticos en la vida de las poblaciones nativas como la de Guatemala, y fue tan grande la ganancia del despojo, es decir, para quienes gozaban de este, que sus formas se han reestructurado en los siglos posteriores, llegando sus consecuencias hasta nuestros días.

En la actualidad, aunque exista una nueva y vasta legislación sobre derechos humanos y estén protegidos por leyes internacionales, la relación con el respeto y la práctica de estos es por lo general, muy dispareja, mucho del discurso del tema, se queda en eso, en perorata oral y escrita, pero con poca práctica, vivencias, faltas de respeto y ausencia de reconocimiento. Por ejemplo, las vulnerabilidades de la ciudadanía en Guatemala son consecuencia de esa estructura formada a lo largo del siglo XIX, conocida como la ciudadanía activa de segunda clase.

Taracena (2002), señala que la condición étnica era una razón para crear dicha ciudadanía, pues dejaba a los indígenas en una situación subalterna, así quedaba más que evidente que "ser guatemalteco era sinónimo de ser ciudadano no-indígena, entendido en términos más culturales que biológicos" (Tomo I, p. 177).

Por lo tanto, ha resultado muy difícil que en la construcción de los nuevos discursos sobre ciudadanía universal y reconocimiento de todos los derechos de las personas, la legislación escrita y promulgada desde la institucionalidad, pueda borrar del imaginario social las prácticas y las leyes que promovieron (después de los tres siglos de Colonia) la formación de nuevas repúblicas basadas en la segregación, el racismo, la discriminación y la concepción de ciudadanos diferentes, en donde el concepto de ciudadanía estaba estrechamente ligado a lo urbano, en oposición a lo indio y a lo rural.

Es así como en los diferentes momentos de la historia de Guatemala en la postindependencia vamos encontrando una ciudadanía que exigía políticas de Estado que no se vincularan a la integración de la población, resaltando los valores occidentales de la población y acentuando las contradicciones indio/ladino.

Todo este discurso y sus prácticas, la institucionalidad, las políticas nacionales y la visión de los funcionarios públicos, así como de los diferentes grupos de la población, fueron reconocidos como parte de la modernidad política, lo que perpetuó la segregación, la invisibilización y la estereotipación del indígena.

Este tipo de ciudadanía diferenciada, que normó las relaciones de poder en Guatemala, fue seguida y fomentada por otros órdenes en el sistema político, económico y social de la nación, como el sistema religioso, el educativo, la maquinaria administrativa del país y por los distintos funcionarios civiles o militares. Por lo que además, el discurso era repetido por las élites, los ladinos, las clases urbanas y demás grupos sociales que veían en el indígena el atraso del pueblo.

Cuando Hinkelammert (2002), habla de la ambivalencia de los derechos humanos y lanza conceptos como el de agresividad humanitaria, el de violación de los derechos de aquellos que los violan, el de inversión de los derechos humanos o el de aniquilación de los derechos humanos, y luego lanza la idea de cómo la sangre derramada por algunos, no deja mancha, nos hace pensar en cómo en este proceso de justificaciones son parte a la vez de las violaciones a los derechos humanos, o 
incluso de inversiones en las que las víctimas resultan ser los culpables y los victimarios terminan de inocentes.

Entonces, es cuestionable el asunto de la legitimidad, pues la misma elaboración de la legalidad de los derechos y los no derechos ha pasado por la vista de quienes son violadores y legalizan sus acciones, legitimando sus pensamientos y desvalorizando la idea de que estén violando los derechos humanos, pues naturalizan sus actos.

Las formas históricas de naturalizar y normar el racismo fueron más que evidentes, bajo las tesis del indio sin alma o de la ciudadanía de segunda categoría en la historia de Guatemala, fueron dotando de culpas al indio que retrasaba el desarrollo de los civilizados, que hacía que los que ostentaban una posición superior, pudiesen castigarlo por rebelde. ¿Por qué? Por una situación de inferioridad legítima. Como dice Hinkelammert (2002), "la inversión de los derechos humanos pasa por toda la interpretación liberal de esos derechos" (p. 23).

Para la teoría moderna de derechos humanos, esa libertad no le da licencia, aunque haya sido naturalizada. Sin embargo, entendiendo el contexto de la historia en Guatemala, en la cual era válido castigar a un culpable, que por su origen étnico no tenía derechos, haciéndose ejecutor de una ley natural (concepto de Locke, citado por Hinkelammert), esta famosa inversión de derechos humanos podía explicarse bien.

En la lógica de que el victimario no es culpable, aunque haya hecho todo tipo de daños al otro, $\mathrm{y}$ justificarlos porque el otro tiene que pagar porque está pagando por los daños que por subordinado le causa al primero, se encuentra lo que Hinkelammert (2002), llama citando a Locke, apropiarse de los bienes o los servicios del culpable.

Según lo anterior, lo primero que debemos analizar es la situación de la deshumanización del ser indígena y todas las vulnerabilidades sujetas a ese proceso de deshumanización, en lo que nos explica el derecho natural, en la idea de que el otro ha sido considerado, en su pasado, uno que aún marca las estructuras actuales de esta sociedad, un esclavo por naturaleza, que es culpable de sus diferencias y por lo tanto hubo un momento en el que fue considerado un ser sin alma. ${ }^{6}$

$\overline{6}$ Haciendo alusión al proceso de conquista y a la colonización.
En segundo lugar, como señala Rodríguez (2009), ¿Qué debemos hacer para que los derechos humanos sean una posibilidad real de mejoramiento para la humanidad? Pensando en el presente, es decir, que no solo sean un discurso de buena voluntad, sino una forma de reivindicar, recuperar y mantener la dignidad del ser humano y la protección tanto del Estado como de otros actores sociales. Si observamos las crisis de la coyuntura actual en Guatemala, diremos que esto aún es un fracaso para la teoría de derechos humanos y que análisis como los que hacemos en este texto, podrían ayudarnos a repensar el presente.

La vulnerabilidad social, económica y política, ha sido por lo tanto, parte de la historia de muchos grupos de población, las herencias coloniales y los procesos por medio de los que se formó el Estado-Nación en la Guatemala del siglo XIX, definieron las relaciones sociales de poder, de desarrollo y de despojo, partiendo inicialmente del despojo de la humanidad, el cual fue seguido de otras formas de despojo que sirvieron para consolidar esas formas de poder y de relación, de exclusión y de segregación.

\section{El despojo de la humanidad de las personas, análisis en la historia de Guatemala}

Severo Martínez (1990), en el capítulo VIII del libro La patria del criollo, titulado "La colonia y nosotros", afirmaba que:

Los grandes problemas de fondo de la actualidad guatemalteca (los que hemos llamado raíces de la problemática actual) son realidades coloniales que se han mantenido por mucho tiempo después de la emancipación sin alterar su esencia a pesar de ciertos desarrollos (...) En rigor, aunque diversos fenómenos de superficie parezcan indicar otra cosa, la verdad es que las dictaduras cafetaleras fueron la realización plena y radicalizada de la patria criolla (p. 588-589)

Concentrándonos en la temática que nos interesa, podríamos asegurar que si precisamente la base de tales realidades coloniales que podemos considerar como los ejes de las problemáticas de Guatemala durante los posteriores siglos, parten en efecto de una problemática, 
base, fundamental, que fue la de deshumanización de las personas, este ha de ser el primer eje de análisis sobre el cual debemos concentrarnos, para encontrar (como dice Martínez Peláez), las raíces de la problemática actual.

Es fundamental, cuando se analiza la historia guatemalteca, mantener presente la pervivencia del poder económico y material, así el peso ideológico que social y políticamente ha marcado el control de las élites económicas en la sociedad, consolidadas alrededor de relaciones de parentesco y núcleos de poder como en el caso del racismo, el despojo material, la legislación que permitió su superioridad por sobre los demás, el control del Estado, o, como señala Casaús (2007), ${ }^{7}$ la conformación de estructuras de larga duración. ${ }^{8}$

En la historia de Guatemala, entender de manera integral, científica y crítica, las formas en que las élites y el Estado se han apoderado de la tierra y del indio (de su trabajo), desde el momento de la conquista, y, luego comprender cómo lograron mantener el control sobre estos dos elementos en el período de la independencia, en la formación de la Nación o a lo largo del siglo XX, es una cuestión básica; pues en la constitución de diversos mecanismos de control, dominación y mantenimiento del status quo establecido, es que se fueron conformando estructuras de poder de larga duración, el núcleo político ha estado históricamente, cimentado en el despojo, en la desigual repartición de la riqueza y en el racismo.

Esto último nos interesa, para el análisis de este documento, pues el despojo de la humanidad de las personas permitió su trato y concepción como animales, como seres desprovistos de cualquier estima o

7 En el texto Linaje y racismo de Marta Casaús (2007), se señala que para establecerse como estructuras de poder de larga duración en Centroamérica, las redes familiares han estado ligadas por medio de cinco factores que les han conferido homogeneidad y unidad: a) las alianzas a través del matrimonio; b) las alianzas a través de los negocios; c) la proximidac geográfica y el factor socioracial; d) la participación en asociaciones políticas, religiosas o socioculturales; y, e) la formación de sus propios intelectuales orgánicos. (p. 8)

8 Estructuras de larga duración a través de redes familiares, ocupando espacios de legitimidad, manipulando diversas coyunturas políticas a su favor, gestando y viviendo diversa estrategias de dominación asícomo resguardando y acrecentando grandes sumas de capita los procesos de corrupción que les permiten asegurarse seguir manteniendo el control y el poder económico, político y social. respeto, como no merecedores de la libertad o de la ciudadanía, como no personas.

El genocidio y la violencia extrema que Guatemala vivió en la segunda mitad del siglo XX (en tiempos del conflicto armado interno), es parte de toda esta configuración del racismo, en la sociedad moldeada a imagen y semejanza del despojo, en la cultura que decidieron las élites del país, con altos componentes de diferencia y exclusión, con una herencia europea y occidentalizada, que los marcó con una fuerte carga de conservadurismo religioso y falsos intentos de modernización.

Cuando en el siglo XXI, nos encontramos con élites económicas y políticas que son descendientes de ese Estado oligárquico que se consolidó a finales del siglo XIX, es que podemos entender el fracaso de las formas democráticas que supuestamente vivimos hoy, ya que muchas de estas solamente han llegado como parte de la reorganización del Estado ante las exigencias del modelo internacional o ante la teoría política moderna, pero siguen impregnadas de prácticas y discursos populistas, de formas de poder ocultas que mantienen intactos los elementos que han marcado el despojo y el racismo en Guatemala.

Entonces, el racismo es además de un factor estructural para entender la historia de Guatemala, el elemento clave que otros siguen usando para mantener el poder, el despojo, el control y la idea que no todas las vidas valen lo mismo en este país, ni todos tenemos los mismos derechos, pues, aunque la teoría, las leyes y los discursos digan que sí, las condiciones de desarraigo, abandono, de miseria y de muerte ${ }^{9}$ confirman otra situación.

Aunque en la actualidad el estereotipo indio/ladino haya variado, en el imaginario nacional, de manera general, aún no han desaparecido ni el racismo, ni la discriminación como prácticas, para continuar con el despojo y la dominación de unos sobre otros, las formas de cohesión que han demostrado las élites del país, para mantener sus formas de poder económico o político, se revisten de una serie de planteamientos que justifican la condena y la criminalización de los indígenas, de los campesinos, de los grupos a los que socialmente consideran inferiores,

9 Según el último Informe de Desarrollo Humano 2015/2016 (PNUD), el 62,4 de la población vive en pobreza media, el 29,6 en pobreza extrema y el $3,6 \%$ en pobreza extrema. 
principalmente cuando las demandas de estos se relacionan con la defensa del territorio o con la reivindicación política y laboral. ${ }^{10}$

Los actuales asesinatos, persecuciones, amenazas y estructuración de discursos de odio contra las organizaciones indígenas, campesinas y populares de Guatemala, tienen su génesis en los temores por perder el orden y la desigualdad que históricamente, han marcado las relaciones de poder y de producción en el país, por defender la acumulación en pocas manos y la desacumulación de la riqueza en grandes grupos de población.

Manejando el discurso racista que constantemente polariza a la mayoría de los guatemaltecos, se han elaborado discursos en contra de la protesta social y la organización política de los grupos desposeídos, de ahí que quienes se consideran diferentes y superiores al otro, no muestren ningún sentimiento, ni ninguna culpa por fomentar la opresión, la represión o la humillación de quienes consideran inferiores.

La explotación, desde que legal e institucionalmente fueron establecidas: la encomienda, el repartimiento, las reducciones de indios, las leyes de ladinización, los mandamientos, el Reglamento de Jornaleros, las habilitaciones, los pagos por medio de fichas de finca, la Ley contra la Vagancia, la Ley del Boleto de Vialidad, la Ley de Protección a la Finca y todas las demás formas y leyes de trabajo forzado, fue normalizándose dentro de las relaciones sociales, que a lo largo de la historia de Guatemala han justificado la exclusión del indígena por no considerarlo persona, o en todo caso, una con menos derechos.

La institucionalidad educativa, religiosa, cultural o mediática ha fomentado que se mantengan esas condiciones de superioridad, racismo y discriminación, y continúa observándose en todos los niveles y formas de expresión, encubriéndose en situaciones legales, culturales y relacionales, que intentan justificar la continuidad de los estereotipos que marcan la inferioridad del otro, en el nivel racial o culturalmente inferior, considerado diferente y por lo tanto de menor categoría.

10 Analícese el caso de organizaciones como CODECA, CCDA y otras que en los últimos tiempos han tenido que sufrir acoso, persecución, asesinatos, criminalizaciones y toda la maquinaria estatal y social en contra, por representar sus exigencias ante el poder de las élites. Tachándoseles de desestabilizadores.
En el libro Café y campesinos, Castellanos Cambranes (1996), señala que en el siglo XIX era comúnmente observable el doble despojo del campesinado, el de su fuerza de trabajo y el de sus tierras. Revisando la historiografía de la época, que además señala la formación de un nuevo modelo de desarrollo que podría llamarse capitalismo neocolonial (como lo nombró Castellanos Cambranes en 1985), podríamos afirmar acá que lo anterior condujo a un tercer despojo, el de la posibilidad de haber surgido un modelo de desarrollo nacional incluyente, un capitalismo nacionalista propio y sin intervención extranjera, un modelo de desarrollo que no estuviese marcado por el latifundismo y la oligarquía terrateniente.

El Estado finquero, que se desarrolló dentro del modelo liberal oligárquico y la economía de plantación para el mercado agroexportador tuvo éxito en el proceso del despojo, y no solo del iniciado con la conquista, sino del organizado como estructura de larga duración, ayudado de otra serie de factores sociales, culturales, educativos, legales, legitimantes e institucionales, los cuales se han entrelazado para mantener las bases de una sociedad en la que se odia al indígena, al pobre, al que se piensa inferior, a quien se sigue considerando que obstaculiza el desarrollo, atrasa la modernidad y detiene las inversiones del empresariado nacional y extranjero (por no someterse a sus intereses económicos).

Un texto extraído del libro de Taracena (2004), nos ayuda a ilustrar esas condiciones de inferioridad racial, étnica, social, política y cultural en la sociedad guatemalteca:

Con el surgimiento de la lucha armada y la ejecución por parte de los gobiernos militares de la Doctrina de Seguridad Nacional a inicios de la década de 1960, la influencia de los planteamientos indigenistas de SISG y del IIN ${ }^{11}$ fue perdiendo fuerza en el seno del Estado guatemalteco. Así, la nueva forma de integrarlos al "progreso nacional" pasó a ser la propuesta de convertirlos en factores de desarrollo económico por medio del cooperativismo, los proyectos de colonización, la educación bilingüe, el servicio militar masivo, etc. De tal manera, que, al sacarlos de la pobreza, se evitaría que fuesen manipulados por los grupos insurgentes que cuestionaban la legitimidad del régimen anticomunista

11 Seminario de Integración Social Guatemalteca (SISG). Instituto Indigenista Nacional (IIN) 
surgido en 1954. No se modificó, por tanto, la idea principal de seguir considerando que la enorme mayoría de indígenas estaba a la zaga de la civilización (Tomo II, p. 89).

Esta visión clasista del indígena, ha sido parte de toda la construcción de nación, soportando legislaciones, discursos, estereotipaciones, formas de aislamiento, manipulaciones, segregaciones y la existencia estructural del racismo, que vio en el genocidio la solución, y, que sigue viendo en las protestas de los pueblos indígenas, el peligro del reconocimiento, el miedo a tener que aceptar que las defensas son legítimas y que conllevan no solo reconocimiento y respeto, sino reivindicación material y económica, y redistribución adecuada del poder y de la riqueza.

Desde el repartimiento y la encomienda colonial, pasando por las leyes de trabajo forzado del siglo XIX, hasta el genocidio en la segunda mitad del siglo XX y llegando a la protesta social del siglo XXI, los espacios del racismo se han ampliado y reciclado, se han profundizado y ya no solamente, se planifican desde el Estado o desde las élites de poder.

Las estrategias de violencia racista contra el indígena han sido tan fuertes, que hoy en día las relaciones y los discursos se han normalizado en distintos niveles y grupos de población, repitiéndose no solo en las esferas oficiales, sino incluso en la academia, se han difundido muy fuertemente en las redes sociales (fenómeno del siglo XXI) y se han interiorizado en las distintas esferas, discursos y formas de comunicación de la sociedad guatemalteca.

\section{Derechos individuales y colectivos. Las garantías de los derechos de las personas}

Uno de los debates entre derechos individuales y colectivos, como nos dice Rodríguez (2009), consiste en que el cumplimiento de los primeros, podría vulnerar algunos derechos individuales como la propiedad privada, por ejemplo. Sin embargo, analizando las relaciones estructurales de la historia de Guatemala, ese proceso de bienestar común que en algún momento podría poner en riesgo el bienestar y los derechos individuales, es de carácter urgente, principalmente, porque como se ha demostrado, la historia del país se fue construyendo sobre la base del despojo, del racismo y de la exclusión, de la deshumanización del ser humano y de una complejidad histórica que se ha marcado por la normalización de la superioridad de unos sobre la inferioridad de los otros.

El prejuicio de la pureza de sangre definió desde un primer momento el estereotipo de la superioridad racial y social, la trascendencia de la categoría indio se sigue observando en la actualidad, cuando vemos en ciertos grupos de la población el temor porque los pueblos originarios tomen en sus manos el poder y sean conductores de su propio destino.

Martínez (1985), escribía sobre las diferencias que hay entre decir quién es el indio, cómo es el indio o por qué es indio, y, releyendo sus argumentos podemos analizar sobre las condiciones de privilegio que han marcado los discursos de las élites, las mismas que hoy promueven sus derechos por encima de los del indígena, la incomprensión del indio o de la categoría de análisis indio; se siguen observando las prácticas de estereotipación y acciones que fomentan la inferioridad.

Entender estos factores, es entender la necesidad de la búsqueda de los derechos colectivos, los argumentos deben partir de demostrar que los indios siempre fueron humanos, que siempre tuvieron alma (en la concepción cristiana), que no necesitaban de un proceso para ser considerados ciudadanos, que no debieron perder su identidad. La exigencia de los derechos colectivos en Guatemala debe sustentarse en la argumentación de eliminar el racismo como centro de la diferencia y la desigualdad, demostrada, por ejemplo, en los procesos de ladinización del siglo XIX.

Ninguna nación puede obviar las garantías de los derechos colectivos, en Guatemala el indígena fue considerado el obstáculo para el desarrollo de la nación; en esa dualidad indio/ladino, y en la que, por lo general, al indígena se le ha colocado en la parte más baja de la estratificación, en la que lo ladino es la oposición a lo que significa ser indio, los derechos colectivos deben partir de la crítica a ese proceso.

Analizando la genealogía del despojo de la humanidad de grandes grupos de población en Guatemala, e insertándola en la teoría y el discurso de los derechos humanos, se entiende que el derecho de los distintos pueblos indígenas debe gozar de ciertos derechos colectivos, que como dicen Aparicio y Pisarello (2008), deben ser precondición para que se ejerzan otros derechos individuales, así:

Vivir hoy en una sociedad que históricamente ha sido construida sobre la 
La autonomía política y, en general, el derecho a la autodeterminación, constituyen requisitos indispensables para ejercer el derecho a la propia cultura. No en vano, tanto el artículo 1 del Pacto Internacional de Derechos Civiles y Políticos, como del Pacto Internacional de Derechos Económicos, Sociales y Culturales, establece que (...) Todos los pueblos tienen derecho a la autodeterminación. En virtud de este derecho determinan libremente su estatuto político y procuran también por su desarrollo económico, social y cultural (...) Que estos derechos colectivos puedan entrar en conflicto con los derechos de otros individuos o de otros grupos no quiere decir que la propia categoría se deba rechazar (p. 146).

Es acá en donde se deben analizar los intereses en juego, tal y como lo hemos observado en las recientes crisis sociopolíticas y económicas de la nación, pues la protección de los grupos más vulnerables, que son los que inicialmente deben protegerse, ya que han pasado por procesos múltiples de despojo históricamente, son los que menos se han protegido. ${ }^{12}$ Por lo tanto, los derechos colectivos que se sujeten a reivindicar estas graves faltas y otras relacionadas con estas, deberán considerarse derechos fundamentales dentro de los derechos humanos, con las garantías necesarias: institucionales, políticas, supraestatales o autónomas.

Tanto en el ámbito nacional como internacional, las garantías de los derechos humanos deben obedecer el principio de justicia universal para garantizar el respeto, la autodeterminación, la participación, la vida y la igualdad. Los derechos humanos deben promover la satisfacción de todas las necesidades que permitan expandir la autonomía individual y colectiva de las personas, eliminando todas las formas de violencia, desigualdad y los derechos convertidos en privilegios, de los deberes sin derechos.

\section{Conclusión}

La pérdida de la humanidad de las personas es la primera grave violación a sus derechos fundamentales, entender esta situación es razonar que las consecuencias de esta, expresadas en cuestiones políticas, económicas, sociales y culturales, marcarán la vida de una nación de

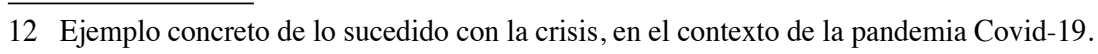

forma trascendente. Ejemplo concreto es lo observado en la Guatemala actual, a raíz de una serie de crisis económicas y sociopolíticas, que se recrudecieron alrededor de una pandemia que nos está afectando aún y en la que lo que menos se ha hecho, es proteger a los grupos más vulnerables y desvalorados históricamente.

En el caso de Guatemala la deshumanización del indio desde el momento de la conquista española, la normalización del racismo y de las relaciones de superioridad/inferioridad, marcaron las formas de poder y de dominación de unos a otros, principalmente, porque estos despojos, que nacieron del despojo de ser humanos, eran la base de otro tipo de robos, como de la tierra, de su fuerza de trabajo o de la posibilidad de ser considerados parte de la patria y de la sociedad que se formaba.

Estas herencias históricas del despojo no han desaparecido, y, en la modernidad de los siglos XX y XXI, se han creado nuevas formas de control, sumisión, desprecio y racismo para controlar los privilegios de unos y el desinterés por los otros.

El despojo en la sociedad guatemalteca ha sido material, político, social, cultural y sobre todo humano. Esto ha afectado gravemente las relaciones de poder, las formas de participación, los procesos de adquisición y repartición de la riqueza y la conformación de una sociedad que no abandonó nunca las condiciones de miseria extrema, de pobreza en general y de racismo.

El racismo como factor determinante en la configuración económica, política y social, ha sido fomentado, mantenido y resguardado, como estructura de larga duración, que necesita de su reproducción en todo nivel y por todos los grupos de la población, pues de él se sujetan las condiciones de riqueza y poder de unos sobre otros, las cuales no se pueden perder.

Esta sociedad se construyó desde el principio de la dominación perpetuada en el prejuicio, a raíz de las formas de resguardar la pureza de sangre y el despojo, en una sociedad que cinco siglos después se siguen manteniendo las relaciones de poder, dominación y control, a partir del mantenimiento de una visión racista, estructurada en varios órdenes y círculos de pobreza y pobreza extrema. 


\section{Bibliografía}

Adorno, R. (1992). Los debates sobre la naturaleza del indio en el siglo XVI: textos y contextos. En: Revista de Estudios Hispánicos. N.1․ 19. ISSN 0378-7974. https://dialnet.unirioja.es/servlet/articulo?codigo=136799

Aparicio, M. y Pisarello, G. (2008). Los derechos humanos y sus garantías: nociones básicas. En: Los derechos humanos en el siglo XXI: continuidad y cambios. España: Huygens Editores.

Casaús, M. (2000). La metamorfosis del racismo en la élite del poder en Guatemala. En: Nueva Antropología, volumen XXVII. México: Asociación Nueva Antropología A. C. https://www.redalyc.org/pdf/159/15905803.pdf

Casaús, M. (2007). Guatemala: linaje y racismo. Tercera edición. Guatemala: F. y G. Editores.

Casaús, M. (2008). Genocidio ¿la máxima expresión del racismo en Guatemala? N. 4 , Cuadernos del presente imperfecto. Guatemala: F. y G. Editores.

Castellanos C. J. (1996). Café y campesinos. Los orígenes de la economía de plantación moderna en Guatemala, 1853-1897. Segunda edición. Guatemala: Editorial Catriel.

CEPAL. (2020). Estudio: Panorama Social de América Latina 2020. Presentación de Aída Bárcena (Secretaria Ejecutiva). Portal en Línea: www.cepal.org

Comisión Nacional de Reparación y Reconciliación -Área de memoria histórica(2009). El despojo de tierras y de territorios: aproximación conceptual. Colombia: Editorial Kimpres Ltda.

Cunill, C. (2011). El indio miserable: nacimiento de la teoría legal en la América colonial del siglo XVI. En: Cuadernos de Intercambio. Año 8. N. 9. 229-248. ISSN: 1659-0139. file:///C:/Users/USUARIO/Downloads/Dialnet-ElIndioMiserable-5089030.pdf

Dardón, R. (2003). El indio: perspectiva histórica. En Revista Estudios N ․ 47, Instituto de Investigaciones Históricas, Antropológicas y Arqueológicas. Escuela de Historia, USAC. Guatemala: Universidad de San Carlos de Guatemala

Galeano, E. (2004). Las venas abiertas de América Latina. Septuagésimo sexta edición. México: Siglo XXI Editores.

Hinkelammert, F. (2002). La inversión de los derechos humanos. El caso de John Locke. En: http://coleccion.uca.edu.sv/franz-hinkelammert/items/show/2040

Mariátegui, J. (2000 [1928]). El problema del indio. En: Siete ensayos de interpretación de la realidad peruana. Argentina: Bibliotecas Rurales Argentinas,
Marxists Internet Archive. En: https://www.marxists.org/espanol/mariateg/1928/7ensayos/02.htm.

Martínez, S. (1990). La patria del criollo. Ensayo de interpretación de la realidad colonial guatemalteca. Décimo primera edición. Guatemala: Ediciones En Marcha.

Martínez, S. (1985). Racismo y análisis histórico en la definición del indio guatemalteco. Investigación para la docencia. Guatemala: Facultad de Ciencias Económicas, USAC.

Peláez, O. -Compilador-. (2008). La patria del criollo, tres décadas después. Guatemala: Editorial Universitaria

PNUD (2016). Informe Nacional de Desarrollo Humano, Guatemala, 2015/2016: más allá del conflicto, luchas por el bienestar. Guatemala: Programa de Naciones Unidas para el Desarrollo.

Rodríguez, J. (2009). Derechos humanos: una aproximación ética. Guatemala: F. y G. Editores

Santos, B. (2014). Derechos humanos, democracia y desarrollo. Bogotá, Colombia: Colección De Justicia.

Taracena, A. (2002). Etnicidad, Estado y Nación en Guatemala, 1808-1944. Tomo I Guatemala: CIRMA.

Taracena, A. (2004). Etnicidad, Estado y Nación en Guatemala, 1944-1985. Tomo II. Guatemala: CIRMA.

UNICEF. (2020). Portal en línea, noticia: "En Guatemala el $49.8 \%$ de los niños sufre desnutrición crónica", María Claudia Santizo, Oficial de Nutrición en UNICEF Guatemala. CIF G 8445108-7-Registro de Fundaciones de competencia estatal. UNICEF: España. https://www.unicef.es/noticia/ en-guatemala-el-498-de-los-ninos-sufre-desnutricion-cronica-maria-claudia-santizo-oficial 